\title{
Association of Kidney-Related Safety Events with Incident Chronic Kidney Disease in Veterans
}

\author{
Helman SR', Stewart PM², Siddiqui T ${ }^{3,4}$, Fink J C ${ }^{1,4}$ \\ and Weiner $\mathbf{S}^{1 *}$ \\ ${ }^{1}$ Department of Medicine, University of Maryland School \\ of Medicine, USA \\ ${ }^{2}$ Pharmaceutical Research Computing, University of \\ Maryland School of Pharmacy, USA \\ ${ }^{3}$ Division of Endocrinology, Diabetes, and Nutrition, \\ University of Maryland School of Medicine, USA \\ ${ }^{4}$ Division of Nephrology, University of Maryland School \\ of Medicine, USA \\ *Corresponding author: Weiner S, Department of \\ Medicine, University of Maryland School of Medicine, 419 \\ West Redwood Street, Suite 620, Baltimore, MD 21201, \\ USA
}

Received: J une 18, 2021; Accepted: J uly 15, 2021; Published: J uly 22, 2021

\begin{abstract}
Objective: The impact of Nonsteroidal Anti-Inflammatory Drugs (NSAID) and iodine-based contrast exposures on developing Chronic Kidney Disease (CKD) is controversial. We examined the association of these exposures with the development of CKD in a Veteran population.

Methods: A retrospective case-control study of 154,448 veterans from the Veterans Affairs (VA) Corporate Data Warehouse (CDW) database between 2005 and 2014 was conducted to assess the association between incident stage 3 CKD with Acute Kidney Injury (AKI), NSAID use, iodine-based contrast exposures, and comorbid conditions. Stepwise logistic regression was used to determine multivariable adjusted Odds Ratios (OR).

Results: The mean age was 59 (SD \pm 13 ), and the median eGFR was 84 (IQR: 73,96 ). AKI was associated with increased odds of CKD (inpatient: OR=3.76, $95 \% \mathrm{Cl}: 3.44,4.11$; outpatient: $\mathrm{OR}=4.73,95 \% \mathrm{Cl}: 4.09,5.46$ ) and demonstrated escalated odds with $>1$ episode (inpatient: OR=5.72, 95\% Cl: 4.71, 6.95; outpatient: OR=8.36, $95 \% \mathrm{Cl}: 6.32,11.06$ ). Months of NSAID prescriptions was associated with CKD, with ORs at $>0-6$ months, $>6-12$ months, and $>12$ months of $1.27(95 \% \mathrm{Cl}: 1.23,1.32), 1.54(95 \% \mathrm{Cl}: 1.46,1.63)$, and $1.69(95 \% \mathrm{Cl}$ : $1.62,1.77)$ respectively. lodine-based contrast exposure was associated with increased odds of CKD, with ORs for 1-2 Computed Tomography (CT) scans, $\geq 3 \mathrm{CT}$ scans, and left heart catheterization of 1.29 (95\% Cl: $1.24,1.35), 1.29$ (95\% Cl: $1.20,1.28)$, and 1.38 (95\% Cl: $1.17,1.63)$ respectively.
\end{abstract}

Conclusion: AKI events, NSAID use, and iodine-based contrast exposures are associated with increased odds for developing stage 3 CKD in veterans.

Keywords: Chronic kidney disease; Acute kidney injury; lodine-based contrast; Nonsteroidal anti-inflammatory drugs

\section{Abbreviations}

AKI: Acute Kidney Injury; CAD: Coronary Artery Disease; CDW: Corporate Data Warehouse; CHF: Congestive Heart Failure; CKD: Chronic Kidney Disease; CKD-EPI: Chronic Kidney Disease Epidemiology Collaboration; CT: Computed Tomography; DM: Diabetes Mellitus; eGFR: Estimated Glomerular Filtration Rates; ESKD: End-Stage Kidney Disease; HTN: Hypertension; LHC: Left Heart Catheterizations; NSAID: Nonsteroidal Anti-Inflammatory Drugs; VA: Veterans Affairs

\section{Introduction}

Chronic Kidney Disease (CKD) affects up to $15 \%$ of the United States population and is responsible for significant morbidity, mortality, and health care costs [1-3]. Patients with other chronic medical conditions, such as hypertension, diabetes, and cardiovascular disease are at increased risk for developing CKD and progressive disease compared to the general population [4-15]. The compounded impact of these chronic medical conditions creates a need to optimize how we manage patients at risk for CKD to minimize morbidity and mortality.

Kidney-associated safety events due to medical care are common and put patients with comorbidities at risk for incident CKD or disease progression [16-18]. Several studies have demonstrated the effects of individual categories of kidney-associated safety events on CKD. Acute kidney injury (AKI) is intimately tied to CKD [19] and shown to be independently associated with both incident CKD and progression to End-Stage Kidney Disease (ESKD) [20-22]. In addition to AKI, use of nephrotoxic agents, including Nonsteroidal Anti-Inflammatory Drugs (NSAIDs) and iodine-based contrast, may impact kidney function and contribute to incident CKD risk. NSAIDs inhibit prostanoid synthesis, which is believed to interfere in both renal and cardiovascular function [23]. Another possible mechanism of NSAIDs' effect on renal function is via AKI, but AKI events secondary to NSAIDs are relatively rare in patients with higher Estimated Glomerular Filtration Rates (eGFRs) [24,25]. Iodine-based contrast can directly affect the kidney through toxic effects on tubular cells and indirectly by affecting kidney hemodynamics [26]. The role contrast agents play in directly causing AKI and CKD is still disputed, and may be affected by the indication for and location of dye administration [26], with arterial infusion possibly carrying a higher risk of kidney damage than venous infusion [27]. Like NSAIDs, AKI secondary to contrast use is now considered relatively rare in patients with higher eGFRs $[26,28]$. In those with existing CKD, contrast 
exposure has been associated with nephropathy and progression to ESKD in small studies $[29,30]$. Less is known about the association of contrast exposures with incident CKD.

How these common kidney-associated safety events affect the development of CKD is unknown. We conducted a case-control study and developed a model to assess associations of AKI events, NSAID use, and iodine-based contrast exposure with incident stage 3 CKD.

\section{Materials and Methods}

\section{Study population}

Data from the nationwide Corporate Data Warehouse (CDW) database was used to conduct a retrospective case-control study (Figure 1). 374,395 patients in the CDW database had a baseline eGFR measurement of $>60 \mathrm{~mL} / \mathrm{min} / 1.73 \mathrm{~m}^{2}$ between June 1,2005 to December 31, 2008. Patients' labs were followed through December 31, 2014. The Chronic Kidney Disease Epidemiology Collaboration (CKD-EPI) equation was used to calculate eGFR. White race was used for $18 \%$ of patients with missing race data since $80 \%$ of veterans on average are white. 243,215 patients with at least 2 eGFR measurements $>60 \mathrm{~mL} / \mathrm{min} / 1.73 \mathrm{~m}^{2}$ and no eGFR measurements $<60 \mathrm{~mL} / \mathrm{min} / 1.73 \mathrm{~m}^{2}$ were available as controls to identify a strict cohort of stable kidney function above stage 3 CKD. 45,347 possible cases were identified and had a minimum of 180 days before their first eGFR $<60 \mathrm{~mL} / \mathrm{min} / 1.73 \mathrm{~m}^{2}$ and two eGFR measures in the stage $3 \mathrm{CKD}$ range that were 90 days apart. The study was approved by the University of Maryland, Baltimore Institutional Review Board and the Baltimore VA Research and Development Committee.

\section{Selection of Cases and Controls}

Cases and controls were matched by time of follow-up with follow-up time defined as the time between the baseline eGFR and either the last available eGFR for controls or the first eGFR of $<60 \mathrm{~mL} / \mathrm{min} / 1.73 \mathrm{~m}^{2}$ for cases. Follow-up times were within 90 days of each other. Cases were assigned to three controls. After available matching, the final cohort included 38,612 cases and 115,836 controls for analysis (Figure 1).

\section{Exposure determination and covariates}

The main exposures included in the analysis were NSAID use, iodine-based contrast exposure, and inpatient and outpatient AKI episodes. Only exposures that occurred during the follow-up time were collected for analysis. Exposure to NSAIDs was determined by the number and months of oral, NSAIDs prescribed at the VA. All intravenous Computed Tomography (CT) studies with contrast, non-cardiac angiograms, and Left Heart Catheterizations (LHC) were used to determine exposure to contrast. All outpatient and inpatient encounters that included the ICD9 codes of 584.x were used to determine AKI events. Baseline comorbidities of Congestive Heart Failure (CHF), Coronary Artery Disease (CAD), hypertension, diabetes, lymphoma, solid organ cancer, and metastatic cancer were determined by ICD 9 codes if they occurred between 180 days preand post- the first eGFR measurement (Supplementary Table 1). Demographics of age, gender, and race were collected as available.

\section{Statistical analysis}

Baseline characteristics and exposures to kidney-related safety events were compared by the mean and standard deviation for normally distributed continuous variables, medians and IQR for continuous variables that were not normally distributed, and/or were divided into categories along with other categorical variables with comparison of percentages. Age was divided into quintiles and eGFR was divided into clinically meaningful categories. Univariate analysis was performed with OR calculations for each risk factor. Stepwise logistic regression with an inclusion p-value of 0.2 and remaining value of 0.1 was conducted. The resulting model was controlled for VA region and follow-up time. Effect modification was evaluated between age and all other covariates, and eGFR with contrast and NSAID exposures. Discrimination was evaluated with a c-statistic and calibration was evaluated with a calibration plot divided into 100 groups. All analyses were performed using SAS Enterprise Guide 7.15 HF8 (SAS Institute, Inc.).

\section{Results}

\section{Baseline characteristics and univariate analysis}

38,612 cases were matched to 115,836 controls by follow-up time, creating comparable mean follow-up times between groups (Table 1). $92 \%$ of controls and $96 \%$ of cases were male. $61 \%, 20 \%$ and $18 \%$ of controls were white, black and race unknown, respectively, versus $63 \%, 17 \%$ and $19 \%$ of cases. All regions of the United States were represented in both groups, with the largest proportion of participants coming from the South. Cases were older (63 IQR: 57, 73 versus 56 IQR: 48,63$)$ and had lower baseline eGFR $\left(71.8 \mathrm{~mL} / \mathrm{min} / 1.73 \mathrm{~m}^{2} \mathrm{IQR}\right.$ : 65.3, 81.4 versus $88.1 \mathrm{~mL} / \mathrm{min} / 1.73 \mathrm{~m}^{2}$ IQR: $\left.77.9,99.1\right)$. Cases were more likely to not receive NSAID prescriptions ( $56 \%$ versus $53 \%$ ), but when they did receive prescriptions, they were more likely to receive more than 5 prescriptions (18\% versus 17\%) or more than 12 months of prescriptions ( $13 \%$ versus $12 \%$ ).

In univariate analyses (Table 2), baseline hypertension, diabetes, $\mathrm{CAD}, \mathrm{CHF}$, lymphoma and solid tumors were associated with an increased odds of incident CKD. Iodine-based contrast exposures and inpatient and outpatient AKI events were associated with an increased odds of incident CKD. Six months or less of NSAID prescriptions and 5 or fewer prescriptions showed a protective association to incident CKD, while more than 12 months of NSAID prescriptions had an increased odds of incident CKD.

\section{Multivariate analysis}

A stepwise multivariate logistic regression model was developed to assess the influence of comorbidities and kidney-related safety events on incident stage $3 \mathrm{CKD}$ (Table 3). The model was adjusted for follow-up time and region of patient care. Lower baseline eGFR was associated with increased odds of incident stage $3 \mathrm{CKD}$. Age interacted with hypertension, diabetes and $\mathrm{CHF}$, with younger ages showing increased odds of incident CKD in the presence of each comorbidity (Figure 2). This effect modification was not present for CAD.

Among kidney-related safety events assessed in the multivariate analysis, inpatient and outpatient AKI showed the strongest correlation with incident stage $3 \mathrm{CKD}$ and demonstrated a significant dose response (Table 3). LHC and intravenous contrast CT scans were both associated with increased odds of incident stage 3 CKD. CT scans did not show a dose response with similar effect sizes between 
Table 1: Baseline Participant Characteristics and Exposures.

\begin{tabular}{|c|c|c|}
\hline Patient Characteristic & Controls $(n=115,836)$ & Cases $(n=38,612)$ \\
\hline Mean Follow-Up (years $\pm S D$ ) & $4.4 \pm 2.4$ & $4.4 \pm 2.3$ \\
\hline \multicolumn{3}{|l|}{ Age (years) } \\
\hline Median (IQR) & $56(48,63)$ & $63(57,73)$ \\
\hline$\leq 50$ & $32,331(28 \%)$ & $2,634(7 \%)$ \\
\hline $50-<58$ & $30,642(27 \%)$ & $7,747(20 \%)$ \\
\hline $58-<67$ & $29,835(26 \%)$ & $12,070(31 \%)$ \\
\hline$\geq 67$ & $23,028(20 \%)$ & $16,161(42 \%)$ \\
\hline \multicolumn{3}{|l|}{ Gender } \\
\hline Male & $106,531(92 \%)$ & $36,949(96 \%)$ \\
\hline Female & $9,305(8 \%)$ & $1,663(4 \%)$ \\
\hline \multicolumn{3}{|l|}{ Race } \\
\hline White & $70,120(61 \%)$ & $24,472(63 \%)$ \\
\hline Black & $22,817(20 \%)$ & $6,581(17 \%)$ \\
\hline Other & $1612(1 \%)$ & $430(1 \%)$ \\
\hline Unknown & $21,287(18 \%)$ & 7,129 (19\%) \\
\hline \multicolumn{3}{|l|}{ Region } \\
\hline Midwest & $19,926(17 \%)$ & $7,317(19 \%)$ \\
\hline South & $49,080(42 \%)$ & $16,752(43 \%)$ \\
\hline West/Pacific & $27,847(24 \%)$ & $8,075(21 \%)$ \\
\hline Northeast & $18,983(16 \%)$ & $6468(17 \%)$ \\
\hline \multicolumn{3}{|l|}{ Baseline eGFR $\left(\mathrm{mL} / \mathrm{min} / 1.73 \mathrm{~m}^{2}\right)$} \\
\hline Median (IQR) & $88(78,99)$ & $72(65,81)$ \\
\hline$\geq 90$ & $53,296(46 \%)$ & $4,797(12 \%)$ \\
\hline $75-90$ & $39,802(34 \%)$ & $9,889(26 \%)$ \\
\hline$<75$ & $22,738(20 \%)$ & $23,926(62 \%)$ \\
\hline Baseline Creatinine (mg/dL $\pm S D)$ & $0.95 \pm 0.16$ & $1.07 \pm 0.15$ \\
\hline \multicolumn{3}{|l|}{ Comorbidities } \\
\hline Coronary Artery Disease & $9,979(9 \%)$ & $7,002(18 \%)$ \\
\hline Congestive Heart Failure & $1,823(2 \%)$ & $1,583(4 \%)$ \\
\hline Diabetes Mellitus & $15,679(14 \%)$ & $10,822(28 \%)$ \\
\hline Hypertension & $47,877(41 \%)$ & $25,473(66 \%)$ \\
\hline Lymphoma & $450(0.4 \%)$ & $208(0.5 \%)$ \\
\hline Solid Organ Cancer & $6,832(6 \%)$ & $3,341(9 \%)$ \\
\hline Metastatic Cancer & $619(0.5 \%)$ & $181(0.5 \%)$ \\
\hline \multicolumn{3}{|l|}{ Angiogram } \\
\hline 0 & $115,332(99.6 \%)$ & $38,383(99.4 \%)$ \\
\hline 1 & $383(0.3 \%)$ & $174(0.5 \%)$ \\
\hline$\geq 2$ & $119(0.1 \%)$ & $55(0.1 \%)$ \\
\hline \multicolumn{3}{|l|}{ CT Scan } \\
\hline 0 & $97,424(84 \%)$ & $31,526(82 \%)$ \\
\hline 2-Jan & $14.071(12 \%)$ & $5,437(14 \%)$ \\
\hline$\geq 3$ & $4,241(4 \%)$ & $1,649(4 \%)$ \\
\hline \multicolumn{3}{|l|}{ Left Heart Catheterization } \\
\hline 0 & $115,341(99.6 \%)$ & $38,274(99.1 \%)$ \\
\hline
\end{tabular}

\begin{tabular}{|c|c|c|}
\hline$\geq 1$ & $495(0.4 \%)$ & $338(0.9 \%)$ \\
\hline \multicolumn{3}{|c|}{ Inpatient AKI } \\
\hline 0 & $114,398(98.8 \%)$ & $36,295(94 \%)$ \\
\hline 1 & $1,230(1 \%)$ & $1,778(5 \%)$ \\
\hline$>1$ & $208(0.2 \%)$ & $539(1 \%)$ \\
\hline \multicolumn{3}{|c|}{ Outpatient AKI } \\
\hline 0 & $115,372(99.6 \%)$ & $37,317(96.6 \%)$ \\
\hline 1 & $384(0.3 \%)$ & $949(2.5 \%)$ \\
\hline$>1$ & $80(0.1 \%)$ & $346(0.9 \%)$ \\
\hline \multicolumn{3}{|c|}{ NSAID Prescriptions } \\
\hline 0 & $61,436(53 \%)$ & $21,488(56 \%)$ \\
\hline 1 & $13,834(12 \%)$ & $3,939(10 \%)$ \\
\hline 3-Feb & $13,745(12 \%)$ & $3,925(10 \%)$ \\
\hline 5-Apr & $7,336(6 \%)$ & $2,341(6 \%)$ \\
\hline$>5$ & $19,485(17 \%)$ & $6,919(18 \%)$ \\
\hline \multicolumn{3}{|c|}{ Months of NSAID Prescriptions } \\
\hline 0 & $61,436(53 \%)$ & $21,488(56 \%)$ \\
\hline$>0-6$ & $32,380(28 \%)$ & $9,200(24 \%)$ \\
\hline$>6-12$ & $8,514(7 \%)$ & $2840(7 \%)$ \\
\hline$>12$ & $13,506(12 \%)$ & $5,084(13 \%)$ \\
\hline
\end{tabular}

Data are presented as $\mathrm{n}(\%)$ unless otherwise indicated. SD = standard deviation $\mathrm{IQR}$ = interquartile range; eGFR = estimated glomerular filtration rate; $\mathrm{AKI}=$ acute kidney injury; NSAID = nonsteroidal anti-inflammatory drug

Table 2: Univariate Odds Ratios of Baseline Characteristics and Exposures.

\begin{tabular}{|c|c|}
\hline Patient Characteristic & Odds Ratio $(95 \% \mathrm{Cl})$ \\
\hline
\end{tabular}

\begin{tabular}{|l|c|}
\hline Age (years) & Ref \\
\hline$\leq 50$ & $3.10(2.96,3.25)$ \\
\hline $50-<58$ & $4.97(4.75,5.19)$ \\
\hline $58-<67$ & $8.61(8.23,9.01)$ \\
\hline$\geq 67$ & \multicolumn{1}{|c|}{ Ref } \\
\hline Gender & $0.52(0.49,0.54)$ \\
\hline Male & Ref \\
\hline Female & $2.76(2.66,2.86)$ \\
\hline Baseline eGFR (mL/min/1.73m²) & $11.69(11.29,12.10)$ \\
\hline$\geq 90$ & $1.51(1.44,1.56)$ \\
\hline $75-90$ & $0.88(0.74,1.03)$ \\
\hline$<75$ & $2.35(2.27,2.43)$ \\
\hline Comorbidities & $2.67(2.50,2.86)$ \\
\hline Coronary Artery Disease & $2.49(2.42,2.56)$ \\
\hline Congestive Heart Failure & $2.75(2.68,2.82)$ \\
\hline Diabetes Mellitus & $1.39,1.64)$ \\
\hline Hypertension & \\
\hline Lymphoma & \\
\hline Solid Organ Cancer & \\
\hline Metastatic Cancer & \\
\hline Angiogram & \\
\hline 0 & \\
\hline
\end{tabular}




\begin{tabular}{|c|c|}
\hline 1 & $1.36(1.13,1.63)$ \\
\hline 2 & $1.39(1.01,1.91)$ \\
\hline \multicolumn{2}{|c|}{ CT Scan } \\
\hline 0 & Ref \\
\hline 2-Jan & $1.19(1.15,1.24)$ \\
\hline$\geq 3$ & $1.17(1.11,1.24)$ \\
\hline \multicolumn{2}{|c|}{ Left Heart Catheterization } \\
\hline 0 & Ref \\
\hline 1 & $2.01(1.79,2.37)$ \\
\hline \multicolumn{2}{|c|}{ Inpatient AKI } \\
\hline 0 & Ref \\
\hline 1 & $4.56(4.23,4.90)$ \\
\hline$>1$ & $8.16(6.95,9.58)$ \\
\hline \multicolumn{2}{|c|}{ Outpatient AKI } \\
\hline 0 & Ref \\
\hline 1 & $7.64(6.78,8.60)$ \\
\hline$>1$ & $13.35(10.47,17.03)$ \\
\hline \multicolumn{2}{|c|}{ NSAID Prescriptions } \\
\hline 0 & Ref \\
\hline 1 & $0.81(0.78,0.85)$ \\
\hline 3-Feb & $0.82(0.79,0.85)$ \\
\hline 5-Apr & $0.91(0.87,0.96)$ \\
\hline$>5$ & $1.02(0.98,1.05)$ \\
\hline \multicolumn{2}{|c|}{ Months of NSAID Prescriptions } \\
\hline 0 & Ref \\
\hline$>0-6$ & $0.81(0.79,0.84)$ \\
\hline$>6-12$ & $0.95(0.91,0.998)$ \\
\hline$>12$ & $1.08(1.04,1.12)$ \\
\hline
\end{tabular}

Odds ratios from univariate analysis are indicated with $95 \%$ Confidence Intervals (CI). Ref: Reference category; eGFR: estimated Glomerular Filtration Rate; AKI: Acute Kidney Injury; NSAID: Nonsteroidal Anti-Inflammatory Drug.

1-2 scans versus $\geq 3$. Non-cardiac angiograms were not found to be significantly associated with CKD in the multivariate analysis. To avoid collinearity, only months rather than number of NSAID prescriptions was included in the multivariate analysis. The odds of incident stage $3 \mathrm{CKD}$ increased with an increasing number of months of NSAID prescriptions. Interaction was not observed between eGFR and CT scan, LHC or months of NSAID prescriptions.

The model demonstrated good discrimination for distinguishing cases of incident CKD with a c-statistic of 0.82 (Figure 3). The calibration plot demonstrated good predictability up to a probability of $70 \%$, after which the model over predicted the probability of incident CKD compared to observed (Figure 4).

\section{Discussion}

The presence of multiple comorbidities along with exposures to safety events can impact the development and progression of CKD. In our case-control study conducted in a large VA population, we found multiple comorbidities, intravenous contrast exposures; NSAID use and AKI events were associated with the development of incident
Table 3: Multivariate Logistic Regression Model.

\begin{tabular}{|c|c|}
\hline Patient Characteristic & Adjusted Odds Ratio (95\% Cl) \\
\hline \multicolumn{2}{|l|}{ Comorbidities } \\
\hline No DM* & Ref \\
\hline $\mathrm{DM}, \leq 50$ & $2.98(2.65,3.36)$ \\
\hline DM, $50-<58$ & $2.60(2.43,2.79)$ \\
\hline DM, $58-<67$ & $2.15(2.03,2.27)$ \\
\hline $\mathrm{DM}, \geq 67$ & $1.48(1.41,1.56)$ \\
\hline No HTN* & Ref \\
\hline HTN, $\leq 50$ & $2.37(2.16,2.59)$ \\
\hline HTN, 50-<58 & $1.91(1.81,2.03)$ \\
\hline HTN, 58-<67 & $1.81(1.72,1.91)$ \\
\hline HTN, $\geq 67$ & $1.58(1.51,1.66)$ \\
\hline No $\mathrm{CHF}^{*}$ & Ref \\
\hline $\mathrm{CHF}, \leq 50$ & $2.81(1.90,4.15)$ \\
\hline $\mathrm{CHF}, 50-<58$ & $1.71(1.41,2.07)$ \\
\hline CHF, 58-<67 & $1.63(1.41,1.89)$ \\
\hline $\mathrm{CHF}, \geq 67$ & $1.35(1.21,1.52)$ \\
\hline CAD & $1.15(1.10,1.19)$ \\
\hline \multicolumn{2}{|l|}{ Baseline eGFR (mL/min/1.73m²) } \\
\hline$\geq 90$ & Ref \\
\hline $75-90$ & $2.54(2.44,2.64)$ \\
\hline$<75$ & $10.54(10.13,10.96)$ \\
\hline \multicolumn{2}{|l|}{ CT Scan } \\
\hline 0 & Ref \\
\hline 2-Jan & $1.29(1.24,1.35)$ \\
\hline$\geq 3$ & $1.29(1.20,1.28)$ \\
\hline \multicolumn{2}{|l|}{ Left Heart Catheterization } \\
\hline 0 & Ref \\
\hline 1 & $1.38(1.17,1.63)$ \\
\hline \multicolumn{2}{|l|}{ Inpatient AKI } \\
\hline 0 & Ref \\
\hline 1 & $3.76(3.44,4.11)$ \\
\hline$>1$ & $5.72(4.71,6.95)$ \\
\hline \multicolumn{2}{|l|}{ Outpatient AKI } \\
\hline 0 & Ref \\
\hline 1 & $4.73(4.09,5.46)$ \\
\hline$>1$ & $8.36(6.32,11.06)$ \\
\hline \multicolumn{2}{|l|}{ Months of NSAID Prescriptions } \\
\hline 0 & Ref \\
\hline$>0-6$ & $1.27(1.23,1.32)$ \\
\hline$>6-12$ & $1.54(1.46,1.63)$ \\
\hline$>12$ & $1.69(1.62,1.77)$ \\
\hline
\end{tabular}

Model was adjusted for region and follow-up time. Multivariate adjusted odds ratios with $95 \%$ Confidence Interval $(95 \% \mathrm{Cl})$ are indicated. Ref: Reference category; AKI: Acute Kidney Injury; eGFR: estimated Glomerular Filtration Rate; DM: Diabetes Mellitus; HTN: Hypertension; CHF: Congestive Heart Failure; CAD: Coronary Artery Disease; NSAID: Nonsteroidal Anti-Inflammatory Drug. *Baseline DM, HTN and CHF interact with age. 


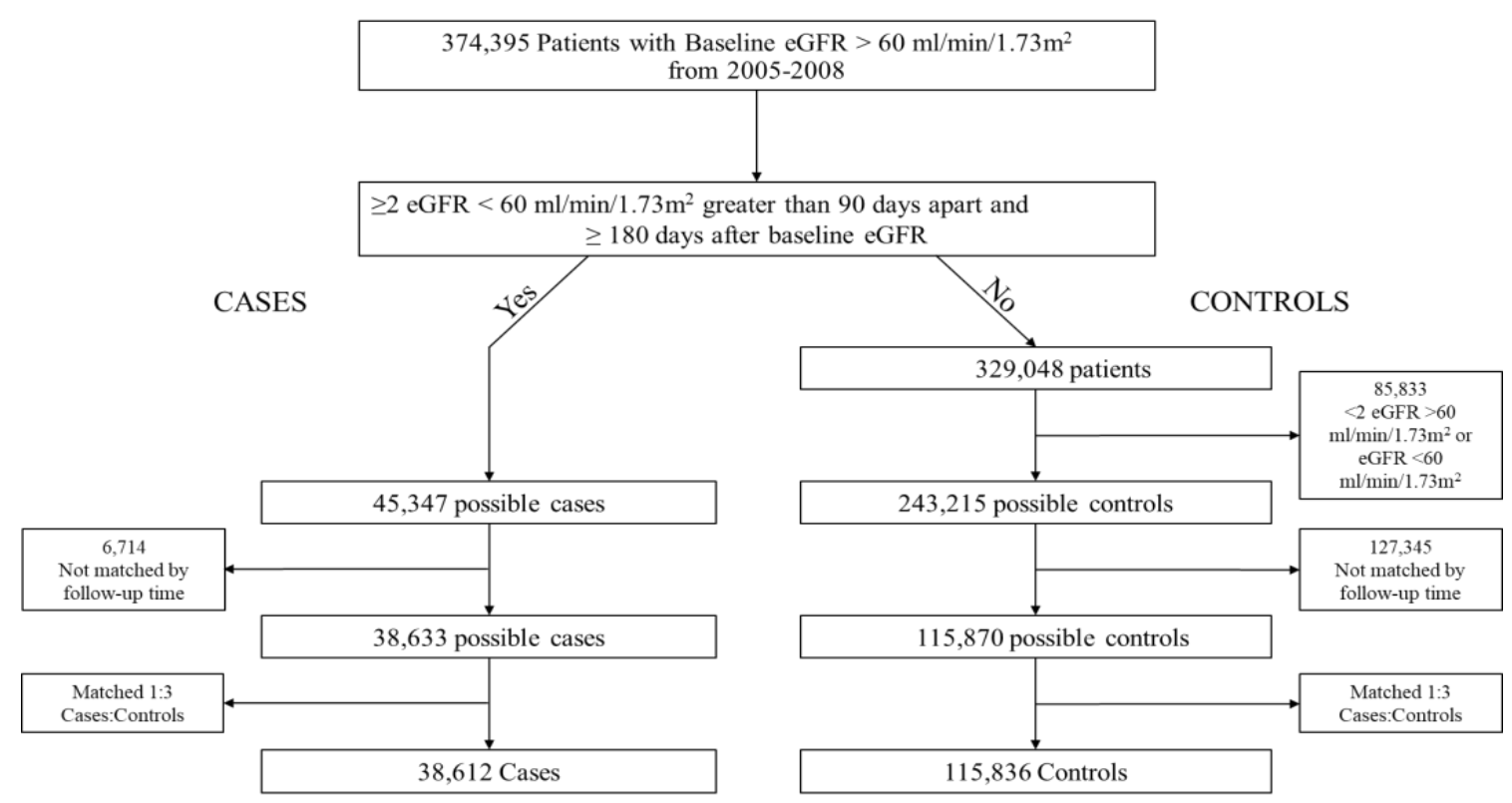

Figure 1: Selection of patients with incident stage 3 chronic kidney disease (cases) and without incident stage 3 chronic kidney disease (controls).

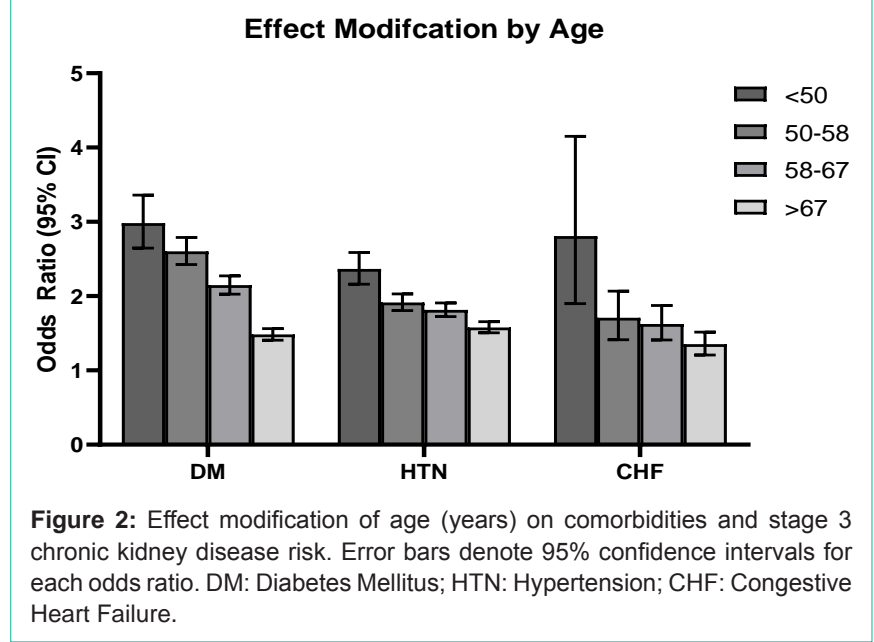

stage 3 CKD. Our study uniquely included a large sample size and regional distribution of participants and evaluated multiple safety exposures simultaneously.

Several generalized prediction models have been developed to understand risks for developing incident $\mathrm{CKD}$, many of which also identify older age, lower baseline eGFR, hypertension, diabetes, CHF, and CAD as risk factors for stage 3 CKD development [4-15]. Hanratty et al. studied 10,420 patients with hypertension in Denver, Colorado. Patients with lower baseline eGFR, older age, diabetes, and vascular disease (composite of CAD, peripheral vascular disease and cerebral vascular disease) had increased risks of incident CKD. CHF was not found to be associated, however only 37 patients had a history of CHF [5]. A study of general community patients from the Atherosclerosis Risk in Communities Study and Cardiovascular Health Study did find CHF associated with CKD at a risk level similar to our findings for our middle-aged groups with an OR of 1.7 in their best-fitted model [11]. They also found age, female gender, diabetes,

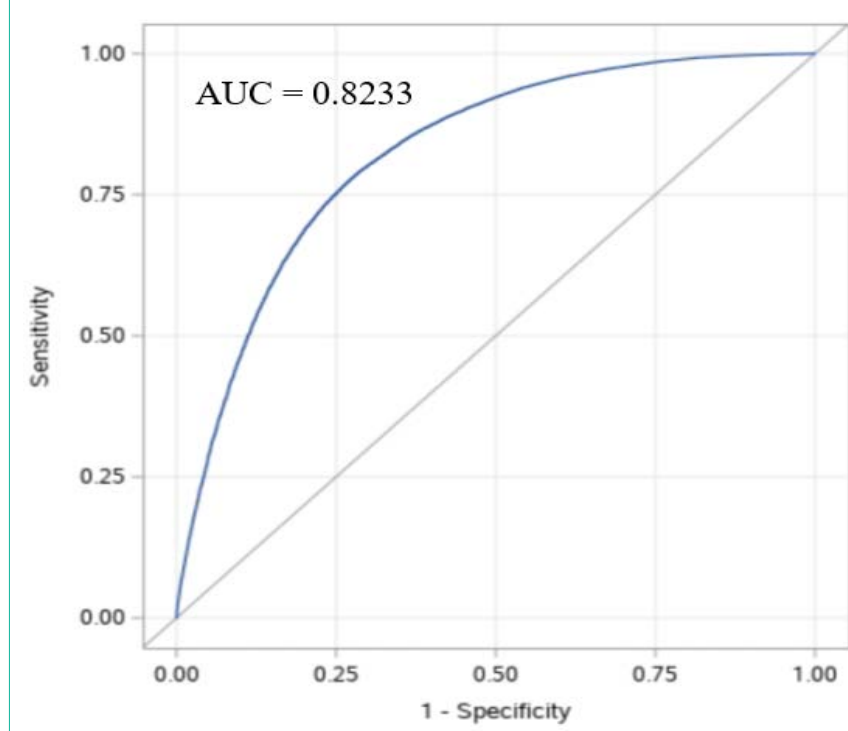

Figure 3: Receiver operating characteristic curve for stage 3 chronic kidney disease model applied to study participants. Model was adjusted for follow-up time and region, and includes age, baseline eGFR, hypertension, diabetes, congestive heart failure, coronary artery disease, inpatient $\mathrm{AKI}$, outpatient AKI, CT scans, left heart catheterizations, and months of non-steroidal antiinflammatory drug prescriptions. AUC: Area under Curve.

hypertension, PVD, anemia, and low high-density lipoprotein levels associated with incident CKD. In the mainly white population of the Framingham study, increased age, lower baseline eGFR, diabetes and smoking were associated with incident CKD [9]. This same population was used to build a prediction model of incident CKD at 10 years that included eGFR, age, diabetes, hypertension and albuminuria [31]. There have also been multiple prediction models in Asian populations that have identified additional risk factors of waist circumference, Body Mass Index (BMI), systolic and diastolic blood pressures, education, C-reactive protein, triglycerides, glucose, and 


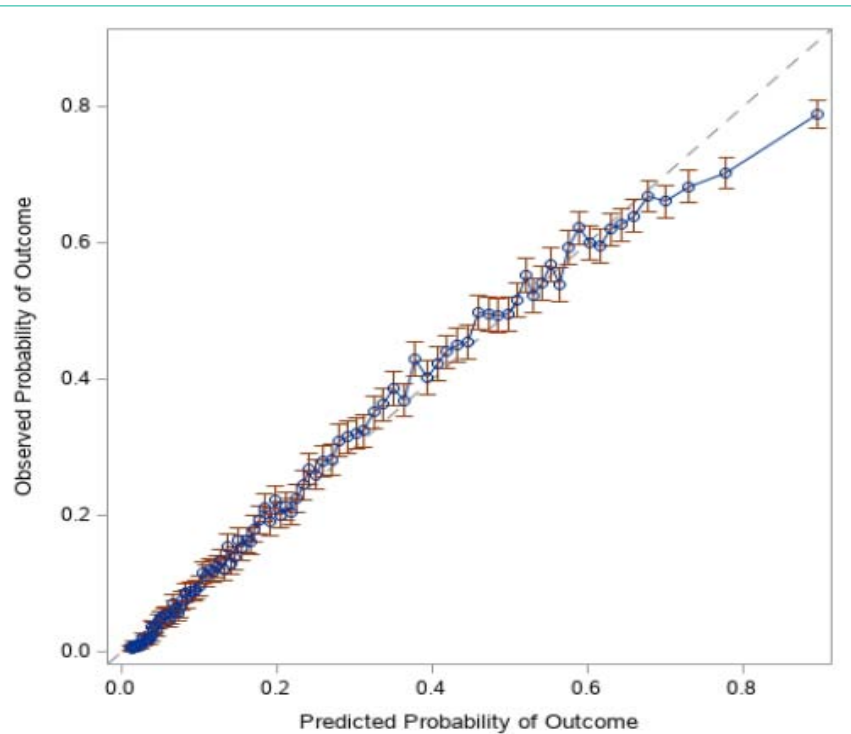

Figure 4: Calibration plot. Predicted probability of incident stage 3 CKD using our model was compared to the observed probability of incident stage $3 \mathrm{CKD}$. Calibration was evaluated with the cohort divided into 100 groups.

uric acid in various models $[7,10,12]$.

Effect modification was not evaluated in the North American studies. Our study showed an interaction between hypertension, diabetes, $\mathrm{CHF}$ and age, with stronger associations between CKD and each comorbidity at a younger age. This likely reflects a survivor bias for younger patients with comorbidities, but also identifies a higher risk population for $\mathrm{CKD}$ that may need more intensive monitoring of kidney function.

In addition to more traditional risk factors, Hao et al.'s study evaluating the use of machine learning methods to predict incident $\mathrm{CKD}$ at 1-year in a Maine population identified risk factors such as number of outpatient visits and radiology tests and different medications, which suggests the possible importance of patient care events in understanding kidney function decline [32].

Inpatient $\mathrm{AKI}$ events are recognized contributors to $\mathrm{CKD}$ development, as shown in other VA studies [20-22,33,34]. A VA cohort comprised of veterans with diabetes who developed AKI during hospitalization was at increased risk for stage $4 \mathrm{CKD}$ development, with each AKI episode approximately doubling the risk [33]. A general VA inpatient study of AKI severity and recovery time conducted by Heung et al. found that mild cases of AKI demonstrated a Hazard Ratio (HR) of 1.43 for the development of CKD within 1 year of admission [20]. Our study is unique in that we assessed both inpatient and outpatient AKI episodes, with outpatient AKI demonstrating a stronger association with developing stage 3 CKD. We did not, however, determine the severity of AKI events, and our control group would be limited to less severe episodes of AKI since the group is defined by all eGFR values remaining $>60 \mathrm{~mL} / \mathrm{min} / 1.73 \mathrm{~m}^{2}$. This may account for the large associations found in our study.

The role of NSAIDs in kidney function decline is still debated. Several studies in healthy populations from the Nurses' Health Study and Physicians' Health Study have not found an association [35-38].
These studies determined NSAID use via questionnaires, which are subject to recall bias [39]. Studies also vary significantly in how they quantify NSAID exposure, with some quantifying by dosage and others quantifying by varying metrics of duration [35-38]. A study based in the United Kingdom that incorporated $>2$ NSAID prescriptions in the preceding 6 months into a model for risk of moderate-severe CKD development noted an increased risk due to NSAID use, with an HR of 1.3 [40]. In a young active military cohort, patients who were prescribed $\geq 7$ doses per month had a $20 \%$ increased risk of CKD, but $<7$ NSAID doses was not associated with an increased risk [41]. Most similar to our findings is the dose response of NSAID exposure in a large hypertensive Taiwanese cohort that found an HR 1.18 for 1 to 89 days of NSAID prescriptions and an HR of 1.32 for $\geq 90$ days of NSAIDs [42]. Other studies have demonstrated that certain NSAIDs, such as ketorolac, oxicams, rofecoxib, ibuprofen, and indomethacin, have increased risk for kidney damage $[25,43]$. Since we did not separate NSAID prescriptions by type, we were unable to assess the effect of specific NSAIDs.

The effect of iodine-based contrast exposure on kidney function is another heavily debated topic and has focused mainly on AKI events following exposures. Decreased eGFR is the only reproducible risk factor for the development of intravenous contrast-induced AKI, which are events directly related to contrast effects on the kidney [44]. Patients with an eGFR greater than $60 \mathrm{~mL} / \mathrm{min} / 1.73 \mathrm{~m}^{2}$ have a risk approaching $0 \%$ for these events, yet may still experience a risk of $5 \%$ for contrast-associated AKI events, which are changes in kidney function of any cause around the timing of contrast exposure $[26,28,44]$. This reflects a growing concern that AKI events may be due to other patient-related factors at the time of contrast exposure, putting into question the direct nephrotoxicity of contrast in patients with better kidney function. A large meta-analysis did not find an increased risk for either AKI or ESKD after exposure to CT contrast compared to CT without contrast [45]. Hinson et al. performed a propensity score matched analysis of emergency room patients who did and did not receive CT contrast and did not find an association between contrast exposure and CKD diagnosis 6 months after exposure [46]. This study only evaluated one contrast exposure, and 6 months may not have been enough time to develop CKD after exposure. We evaluated multiple intravenous contrast exposures over a longer time and found a 30\% increased odds of incident CKD with contrast exposure. The lack of dose response between 1-2 intravenous contrast exposures and $\geq 3$ contrast exposures warrants more evaluation since the lack of a dose response is less supportive of a true correlation. We also did not directly determine if CT studies were associated with the AKI events, however, as AKI events are rare at higher eGFR values, the effect of contrast in this context would likely be reflective of more subtle changes to kidney function.

Arterial contrast exposures have mainly been studied in patients undergoing LHC and incur higher risks of AKI compared to intravenous contrast. Secondary analysis of a randomized control trial of patients comparing LHC to CT angiography for the evaluation of atypical angina revealed $13.2 \%$ of patients undergoing LHC experienced AKI versus $5.6 \%$ of patients undergoing CT angiography [47]. Patients that experienced AKI after LHC were found to be at higher risk of CKD and kidney decline [27,47]. It is still uncertain how much of these effects are due to the contrast itself versus other 
clinical factors surrounding the need for LHC. This concern may reflect our finding that non-cardiac angiograms were not found to be associated with incident CKD in our multivariate analysis, and more research is needed to understand the possible role of contrast from non-cardiac angiograms in kidney function decline.

Our study has several limitations. Since this was a retrospective analysis, causal association between kidney-related safety events and incident stage $3 \mathrm{CKD}$ cannot be determined. There may also be additional confounding from variables that were not evaluated in this analysis such as BMI, smoking and proteinuria. Due to missing race data, there may be misclassification of CKD cases and race could not be evaluated in the final model. Analysis with available race data did not significantly alter model estimates; however, black race carried increased odds of incident CKD in the analysis of participants with race data (Supplementary Table 2). Our conclusions are also limited mainly to men due to the small number of female veterans in this VA cohort. We were also unable to assess the influence of events either occurring prior to the study period or occurring outside the VA that may affect CKD risk. Additionally, the use of ICD-9 codes to identify exposures and comorbidities likely resulted in an underestimation of events and cannot assess the severity of comorbidities. In previous analyses, billing codes for AKI have demonstrated low sensitivity and high specificity, with a tendency to under-report AKI events and typically identify higher severity AKI events [48]. Along similar lines, use of NSAID prescription data does not necessarily indicate total NSAIDs taken by the patient since we could not determine adherence to prescriptions or use of over the counter and external prescriptions. Lastly, we did not validate our findings externally and our findings may not be applicable to patients outside of the VA system.

\section{Conclusion}

CKD is a common condition with many factors that influence its development and progression. Our study demonstrates associations of multiple safety events with incident CKD concurrently, including AKI events, NSAIDs use and contrast exposures. More research is needed to understand the role of preventable safety events in developing CKD and how to best manage exposures to these events to prevent kidney function decline. Preventative efforts may be most beneficial in younger patients with CHF, diabetes, and hypertension.

\section{Acknowledgements}

Funding was provided by Pharmaceutical Research Computing, University of Maryland School of Pharmacy, USA.

\section{References}

1. Saran R, Robinson B, Abbott KC, Agodoa LYC, Bragg-Gresham J, Balkrishnan R, et al. US Renal Data System 2018 Annual Data Report: Epidemiology of Kidney Disease in the United States. Am J Kidney Dis Off J Natl Kidney Found. 2019; 73: A7-A8.

2. Webster AC, Nagler EV, Morton RL, Masson P. Chronic Kidney Disease. The Lancet. 2017; 389: 1238-1252.

3. Murphy D, McCulloch CE, Lin F, Banerjee T, Bragg-Gresham JL, Eberhard MS, et al. Trends in prevalence of chronic kidney disease in the United States. Ann Intern Med. 2016; 165: 473-481.

4. Fox CS, Larson MG, Leip EP, Meigs JB, Wilson PWF, Levy D. Glycemic Status and Development of Kidney Disease: The Framingham Heart Study. Diabetes Care. 2005; 28: 2436-2440.

5. Hanratty R, Chonchol M, Miriam Dickinson L, Beaty BL, Estacio RO,
MacKenzie TD, et al. Incident chronic kidney disease and the rate of kidney function decline in individuals with hypertension. Nephrol Dial Transplant. 2010; 25: 801-807.

6. Gosmanov AR, Lu JL, Sumida K, Potukuchi PK, Rhee CM, Kalantar-Zadeh $\mathrm{K}$, et al. Synergistic Association of Combined Glycemic and Blood Pressure Level with Risk of Complications in US Veterans with Diabetes. J Hypertens. 2016; 34: 907-913.

7. Chia YC, Ching SM. Hypertension and the development of New onset chronic kidney disease over a 10 year period: a retrospective cohort study in a primary care setting in Malaysia. BMC Nephrol. 2012; 13: 173.

8. Burrows NR, Vassalotti JA, Saydah SH, Stewart R, Gannon M, Chen S-C, et al. Identifying High-Risk Individuals for Chronic Kidney Disease: Results of the CHERISH Community Demonstration Project. Am J Nephrol. 2018; 48: 447-455.

9. Fox CS, Larson MG, Leip EP, Culleton B, Wilson PWF, Levy D. Predictors of New-Onset Kidney Disease in a Community-Based Population. JAMA. 2004; 291: 844-850.

10. Chien K-L, Lin H-J, Lee B-C, Hsu H-C, Lee Y-T, Chen M-F. A Prediction Model for the Risk of Incident Chronic Kidney Disease. Am J Med. 2010; 123: 836-846.e2.

11. Kshirsagar AV, Bang H, Bomback AS, Vupputuri S, Shoham DA, Kern LM, et al. A Simple Algorithm to Predict Incident Kidney Disease. Arch Intern Med. 2008; 168: 2466-2473.

12. Wen J, Hao J, Zhang Y, Cao K, Zhang X, Li J, et al. Risk scores for predicting incident chronic kidney disease among rural Chinese people: a village-based cohort study. BMC Nephrol. 2020; 21.

13. Whaley-Connell AT, Sowers JR, Stevens LA, McFarlane SI, Shlipak MG, Norris KC, et al. CKD in the United States: Kidney Early Evaluation Program (KEEP) and National Health and Nutrition Examination Survey (NHANES) 1999-2004. Am J Kidney Dis. 2008; 51: S13-S20.

14. McCullough PA, Li S, Jurkovitz CT, Stevens LA, Wang C, Collins AJ, et al. CKD and Cardiovascular Disease in Screened High-Risk Volunteer and General Populations: The Kidney Early Evaluation Program (KEEP) and National Health and Nutrition Examination Survey (NHANES) 1999-2004. Am J Kidney Dis. 2008; 51: S38-S45.

15. Taal MW, Brenner BM. Predicting initiation and progression of chronic kidney disease: Developing renal risk scores. Kidney Int. 2006; 70: 1694-1705.

16. Chapin E, Zhan M, Hsu VD, Seliger SL, Walker LD, Fink JC. Adverse Safety Events in Chronic Kidney Disease: The Frequency of "Multiple Hits". Clin J Am Soc Nephrol CJASN. 2010; 5: 95-101.

17. Weir MR, Fink JC. Safety of medical therapy in patients with chronic kidney disease and end-stage renal disease. Curr Opin Nephrol Hypertens. 2014; 23: $306-313$

18. Seliger SL, Zhan M, Hsu VD, Walker LD, Fink JC. Chronic Kidney Disease Adversely Influences Patient Safety. J Am Soc Nephrol JASN. 2008; 19: 2414-2419

19. Chawla LS, Eggers PW, Star RA, Kimmel PL. Acute Kidney Injury and Chronic Kidney Disease as Interconnected Syndromes. N Engl J Med. 2014; 371: $58-66$

20. Heung M, Steffick DE, Zivin K, Gillespie BW, Banerjee T, Hsu C, et al. Acute Kidney Injury Recovery Pattern and Subsequent Risk of CKD: An Analysis of Veterans Health Administration Data. Am J Kidney Dis. 2016; 67: 742-752.

21. Jones J, Holmen J, De Graauw J, Jovanovich A, Thornton S, Chonchol M. Association of Complete Recovery From Acute Kidney Injury With Incident CKD Stage 3 and All-Cause Mortality. Am J Kidney Dis. 2012; 60: 402-408.

22. Coca SG, Singanamala S, Parikh CR. Chronic kidney disease after acute kidney injury: a systematic review and meta-analysis. Kidney Int. 2012; 81: 442-448.

23. Cabassi A, Tedeschi S, Perlini S, Verzicco I, Volpi R, Gonzi G, et al. Nonsteroidal anti-inflammatory drug effects on renal and cardiovascular function: from physiology to clinical practice. Eur J Prev Cardiol. 2020; 27: 850-867. 
24. Huerta C, Castellsague J, Varas-Lorenzo C, García Rodríguez LA Nonsteroidal anti-inflammatory drugs and risk of ARF in the general population. Am J Kidney Dis. 2005; 45: 531-539.

25. Winkelmayer WC, Waikar SS, Mogun H, Solomon DH. Nonselective and Cyclooxygenase-2-Selective NSAIDs and Acute Kidney Injury. Am J Med. 2008; 121: 1092-1098.

26. Mehran R, Dangas GD, Weisbord SD. Contrast-Associated Acute Kidney Injury. N Engl J Med. 2019; 380: 2146-2155.

27. James MT, Ghali WA, Tonelli M, Faris P, Knudtson ML, Pannu N, et al. Acute kidney injury following coronary angiography is associated with a long-term decline in kidney function. Kidney Int. 2010; 78: 803-809.

28. McCullough PA, Adam A, Becker CR, Davidson C, Lameire N, Stacul F, et al. Risk Prediction of Contrast-Induced Nephropathy. Am J Cardiol. 2006; 98: 27-36.

29. Ellis $\mathrm{JH}$, Khalatbari S, Yosef $\mathrm{M}$, Cohan $\mathrm{RH}$, Davenport MS. Influence of Clinical Factors on Risk of Contrast-Induced Nephrotoxicity From IV lodinated Low-Osmolality Contrast Material in Patients With a Low Estimated Glomerular Filtration Rate. Am J Roentgenol. 2019; 213: W188-W193.

30. Lim E, Jang J-H, Yoon D, Min Y-G, Kim H-H. Does Exposure to Computed Tomography Contrast Media Increase Risk of End-Stage Renal Disease? Med Sci Monit Int Med J Exp Clin Res. 2020; 26: e921303-1-e921303-7.

31. O'Seaghdha CM, Lyass A, Massaro JM, Meigs JB, Coresh J, D'Agostino RB et al. A Risk Score for Chronic Kidney Disease in the General Population. Am J Med. 2012; 125: 270-277

32. Hao S, Fu T, Wu Q, Jin B, Zhu C, Hu Z, et al. Estimating One-Year Risk of Incident Chronic Kidney Disease: Retrospective Development and Validation Study Using Electronic Medical Record Data From the State of Maine. JMIR Med Inform. 2017; 5

33. Thakar CV, Christianson A, Himmelfarb J, Leonard AC. Acute Kidney Injury Episodes and Chronic Kidney Disease Risk in Diabetes Mellitus. Clin J Am Soc Nephrol CJASN. 2011; 6: 2567-2572.

34. Soto K, Campos P, Pinto I, Rodrigues B, Frade F, Papoila AL, et al. The risk of chronic kidney disease and mortality are increased after communityacquired acute kidney injury. Kidney Int. 2016; 90: 1090-1099.

35. Curhan GC, Knight EL, Rosner B, Hankinson SE, Stampfer MJ. Lifetime Nonnarcotic Analgesic Use and Decline in Renal Function in Women. Arch Intern Med. 2004; 164: 1519-1524.

36. Kurth T, Glynn RJ, Walker AM, Rexrode KM, Buring JE, Stampfer MJ, et al. Analgesic use and change in kidney function in apparently healthy men 1 1The sponsor of the study had no role in study design, data collection, data analysis, data interpretation, or the writing of the manuscript. Am J Kidney Dis. 2003 ; 42 : 234-244
37. Rexrode KM, Buring JE, Glynn RJ, Stampfer MJ, Youngman LD, Gaziano JM. Analgesic Use and Renal Function in Men. JAMA. 2001; 286: 315-321.

38. Agodoa LY, Francis ME, Eggers PW. Association of Analgesic Use with Prevalence of Albuminuria and Reduced GFR in US Adults. Am J Kidney Dis. 2008; 51: 573-583

39. McLaughlin JK, Lipworth L, Chow W-H, Blot WJ. Analgesic use and chronic renal failure: A critical review of the epidemiologic literature. Kidney Int. 1998; 54: $679-686$

40. Hippisley-Cox J, Coupland C. Predicting the risk of Chronic Kidney Disease in Men and Women in England and Wales: prospective derivation and external validation of the QKidney ${ }^{\circledR}$ Scores. BMC Fam Pract. 2010; 11: 49.

41. Nelson DA, Marks ES, Deuster PA, O'Connor FG, Kurina LM. Association of Nonsteroidal Anti-inflammatory Drug Prescriptions With Kidney Disease Among Active Young and Middle-aged Adults. JAMA Netw Open. 2019; 2 : e187896-e187896

42. Hsu Chih-Cheng, Wang Hongiian, Hsu Yueh-Han, Chuang Shao-Yuan, Huang Ya-Wen, Chang Yu-Kang, et al. Use of Nonsteroidal Anti-Inflammatory Drugs and Risk of Chronic Kidney Disease in Subjects With Hypertension. Hypertension. 2015; 66: 524-533.

43. Ingrasciotta Y, Sultana J, Giorgianni F, Fontana A, Santangelo A, Tari DU, et al. Association of Individual Non-Steroidal Anti-Inflammatory Drugs and Chronic Kidney Disease: A Population-Based Case Control Study. PLoS ONE. 2015; 10

44. Davenport MS, Perazella MA, Yee J, Dillman JR, Fine D, McDonald RJ, et al. Use of Intravenous lodinated Contrast Media in Patients with Kidney Disease: Consensus Statements from the American College of Radiology and the National Kidney Foundation. Radiology. 2020; 294: 660-668.

45. Aycock RD, Westafer LM, Boxen JL, Majlesi N, Schoenfeld EM, Bannuru RR. Acute Kidney Injury After Computed Tomography: A Meta-analysis. Ann Emerg Med. 2018; 71: 44-53.e4.

46. Hinson JS, Ehmann MR, Fine DM, Fishman EK, Toerper MF, Rothman $\mathrm{RE}$, et al. Risk of Acute Kidney Injury After Intravenous Contrast Media Administration. Ann Emerg Med. 2017; 69: 577-586.e4

47. Schönenberger E, Martus $P$, Bosserdt M, Zimmermann E, Tauber R, Laule $M$, et al. Kidney Injury after Intravenous versus Intra-arterial Contrast Agent in Patients Suspected of Having Coronary Artery Disease: A Randomized Trial. Radiology. 2019; 292: 664-672.

48. Grams ME, Waikar SS, MacMahon B, Whelton S, Ballew SH, Coresh J. Performance and Limitations of Administrative Data in the Identification of AKI. Clin J Am Soc Nephrol. 2014; 9: 682-689. 